\title{
Adhesion forces promote transcription
}

The mechanisms that link mechanical forces to cellular responses, which are important during development and disease, are poorly understood. In Science, Benham-Pyle et al. now report that mechanical strain applied to quiescent epithelial cells activates the transcription factors YAP1 and $\beta$-catenin in a cadherin-dependent manner, and that this promotes cell cycle entry.

Cadherins form cell-cell junctions through interactions between their extracellular domains, and their cytoplasmic domains are linked to the actin cytoskeleton through $\alpha$-catenin and $\beta$-catenin. This junctional complex regulates growth signalling by sequestering $\beta$-catenin and the Hippo pathway transcription factor YAP1
C6

mechanical

forces activate

YAP 1 - and

$\beta$-catenin-

mediated

transcriptional

activity at

different times

g in the cytoplasm. Using a monolayer of quiescent kidney epithelial cells to which varying stretching forces could be applied, the authors examined whether cadherin-mediated adhesion also regulates YAP 1 and $\beta$-catenin in response to mechanical forces. Mechanical strain induced cell cycle re-entry and progression into the $S$ phase of the cell cycle. In the absence of mechanical strain, YAP1 was found in the cytoplasm and cortex and $\beta$-catenin was found at cell-cell junctions; both moved to the nucleus in response to strain, albeit with different kinetics. YAP1 was detected in the nucleus within 1 hour of strain application, and nuclear levels increased until 6 hours post-strain before decreasing, whereas $\beta$-catenin was in the nucleus from 6 hours and up to 24 hours after strain application. Their transcriptional activity corresponded with their nuclear localization, indicating that mechanical forces activate YAP1- and $\beta$-catenin-mediated transcriptional activity at different times.

Next, the authors found that activation of YAP1 and $\beta$-catenin occurred independently of each other. YAP1 transcriptional activity was required for cell cycle re-entry but was not required for $\beta$-catenin activation. Moreover, $\beta$-catenin transcriptional activity was not required for YAP1 function and cell cycle re-entry but was required for progression into the $\mathrm{S}$ phase.

To test the role of E-cadherin in regulating the response to mechanical strain, a mutant version with a truncated non-functional extracellular domain, but with a normal cytoplasmic domain, was expressed in the epithelial cells forming the monolayer. YAP1 was localized to the nucleus and cells entered G1 whether or not force was applied, but nuclear levels of $\beta$-catenin remained low and cells did not progress to the $S$ phase. Thus, extracellular E-cadherin engagement is required to sequester YAP1 and to mediate strain-induced nuclear accumulation of $\beta$-catenin and cell cycle progression.

In summary, this work indicates that cadherin cell-cell junctions transduce external mechanical signals into transcriptional responses, and that YAP1 and $\beta$-catenin transcriptional activity is important for cell cycle entry and progression.

Kim Baumann

ORIGINAL RESEARCH PAPER Benham-Pyle, B.W., Pruitt, B. L. \& Nelson, W. J. Mechanical strain induces $\mathrm{E}$-cadherin-dependent Yap1 and $\beta$-catenin activation to drive cell cycle entry. Science 348, 1024-1027 (2015)

FURTHER READING Iskratsch, T., Wolfenson, H. \& Sheetz, M. P. Appreciating force and shape - the rise of mechanotransduction in cell biology. Nat. Rev. Mol. Cell Biol. 15, 825-833 (2014) 